\title{
INVARIANT RELATIONS
}

BY F. H. MURRAY

1. Introduction. In a preceding paper* a discussion was given of the systems of invariant relations as defined by Poincaré. $\dagger$ The methods employed there required that all the functions appearing in the differential system or in the invariant relations should be analytic. This restriction is not essential, however, and it is the object of the present note to give a corresponding discussion in which the functions appearing are required to possess only a finite number of derivatives. The integration of the differential system by the method of successive approximations of Picard, after a suitable transformation, gives at once the justification for the name "invariant relation."

2. Transformation of the Differential System. Suppose given a differential system

$$
\frac{d x_{i}}{d t}=X_{i}\left(x_{1}, \cdots, x_{n}\right), \quad(i=1, \cdots, n),
$$

and a system of equations

$$
\phi_{k}\left(x_{1}, \cdots, x_{n}\right)=0, \quad(k=1, \cdots, r) .
$$

The functions $X_{i}, \phi_{k}$ are assumed to possess continuous partial derivatives of the first and second orders with respect to all their arguments, $\left|x_{i}-x_{i}{ }^{0}\right|<C$. Assume that the equations

$$
\sum_{i=1}^{n} X_{i} \frac{\partial \phi_{k}}{\partial x_{i}}=0, \quad(k=1, \cdots, r)
$$

* On certain families of orbits with arbitrary masses in the problem of three bodies, Transactions of this Society, vol. 28, pp. 74-77.

$\dagger$ †es Méthodes Nouvelles de la Mécanique Céleste, vol. 1, pp. 45-47. 
are a consequence of the equations (2); the equations (2) are then said to form a system of invariant relations. If the functions $\phi_{k}$ are not independent, but equations (2) are a consequence of a sub-set of these equations, then (3) is also a consequence of the equations of the sub-set, and the discussion can be restricted to the smaller set.

Without loss of generality, therefore, it may be assumed that the equations (2) are independent. It will be assumed also that the determinant

$$
\left|\begin{array}{ccc}
\frac{\partial \phi_{1}}{\partial x_{1}} & \cdots & \frac{\partial \phi_{1}}{\partial x_{r}} \\
\frac{\partial \phi_{r}}{\partial x_{1}} & \cdots & \frac{\partial \phi_{r}}{\partial x_{r}}
\end{array}\right|
$$

does not vanish for $x_{i}=x_{i}{ }^{0}, i=1, \cdots, n$. Under these assumptions the equations

$$
\begin{gathered}
y_{1}=\phi_{1}, \cdots, y_{r}=\phi_{r}, \\
y_{r+1}=x_{r+1}-x_{r+1}^{0}, \cdots, y_{n}=x_{n}-x_{n}{ }^{0}
\end{gathered}
$$

can be solved for $x_{1}, \cdots, x_{n}$, if $\left|y_{i}\right| \leqq a,(i=1, \cdots, n)$, if $a$ is sufficiently small. If transformed functions are indicated by brackets, (1) becomes

$\left\{\begin{array}{l}\frac{d y_{i}}{d t}=\left[\sum_{k=1}^{n} X_{k} \frac{\partial \phi_{i}}{\partial x_{k}}\right]=Y_{i}\left(y_{1}, \cdots, y_{n}\right),(i=1, \cdots, r), \\ \frac{d y_{k}}{d t}=\left[X_{k}\right]=Y_{k}\left(y_{1}, \cdots, y_{n}\right), \quad(k=r+1 \cdots n) .\end{array}\right.$

Now if $y_{1}=y_{2}=\cdots=y_{r}=0$, equations (2) are satisfied, hence (3), and consequently $Y_{1}=Y_{2}=\cdots=Y_{r}=0$, for all values of $y_{r+k}$ such that $\left|y_{r+k}\right|<a$. Or,

$$
Y_{i}\left(0, \cdots, 0, y_{r+1}, \cdots, y_{n}\right)=0,(i=1, \cdots, r) .
$$

3. Integration of the Equations. Consider a differential system

$$
\frac{d y_{i}}{d t}=Y_{i}\left(y_{1}, \cdots, y_{n}\right), \quad(i=1, \cdots, n)
$$


in which the functions $Y_{i}$ satisfy the conditions

$$
\begin{array}{r}
Y_{i}\left(0, \cdots, 0 ; y_{r+1}, \cdots, y_{n}\right)=0, \quad(i=1, \cdots, r), \\
\left|Y_{k}(0, \cdots, 0)\right| \leqq M, \quad\left|\frac{\partial Y_{k}}{\partial y_{i}}\right| \leqq A_{k},(i, k=1, \cdots, n),
\end{array}
$$

in the region

$$
\left|y_{i}\right| \leqq b \text {. }
$$

Suppose $K=\sum_{i=1}^{n} A_{i}$; the system (6) can now be integrated by the method of successive approximations of Picard:

and in general

$$
y_{i}^{\prime}=\int_{0}^{t} Y_{i}(0, \cdots, 0) d t
$$

The series

$$
\begin{aligned}
y_{i}{ }^{(k+1)}=\int_{0}^{t} Y_{i}\left(y_{1}{ }^{(k)}, \cdots, y_{n}{ }^{(k)}\right) d t \\
(i=1, \cdots, n ; k=1,2,3, \cdots) .
\end{aligned}
$$

$$
y_{i}^{\prime}(t)+\left(y_{i}^{\prime \prime}-y_{i}^{\prime}\right)+\cdots+\left(y_{i}^{(k+1)}-y_{i}^{(k)}\right)+\cdots
$$

converges uniformly in $t$ if $t \leqq T$,

$$
T<\frac{1}{K} \log \left(1+\frac{b K}{M}\right) .
$$

By hypothesis,

$$
Y_{i}(0, \cdots, 0)=0, \quad(i=1, \cdots, r)
$$

consequently

Also

$$
y_{i}^{\prime}(t)=0, \quad(i=1, \cdots, r) .
$$

$$
Y_{i}\left(0, \cdots, 0 ; y_{r+1}^{\prime}, \cdots, y_{n}^{\prime}\right)=0, \quad(i=1, \cdots, r),
$$

whence

$$
y_{i}^{\prime \prime}(t) \equiv 0, \quad 0<t \leqq T, \quad(i=1, \cdots, r) .
$$

By the method of induction it is seen at once that every one of the functions $y_{i}{ }^{(k)}(i=1, \cdots, r)$ vanishes identically in $t$, and consequently the sum of the series $y_{i}$ must likewise vanish identically. If the conditions $y_{i}{ }^{0}=0,(i=1, \cdots, r)$, are satisfied, then $y_{i}$ vanishes identically for $t \leqq T$. 
Suppose that after the transformation of $\S 1$, the differential system satisfies the hypotheses stated at the beginning of this paragraph. The equations $\left[\phi_{i}\right]_{t=0}=0$ imply the equations $y_{i}^{0}=0,(i=1, \cdots, r)$, which imply that

$y_{i} \equiv \phi_{i}\left(x_{1}, \cdots, x_{n}\right)=0, \quad 0 \leqq t \leqq T, \quad(i=1, \cdots, r)$

The equations (2) are satisfied, therefore, for all values of $t \leqq T$, and the name "invariant relations", applied to (2), is completely justified.

Dalhousie University

\section{DETERMINATION OF THE NUMBER OF SUB- GROUPS OF AN ABELIAN GROUP}

BY G. A. MILLER

The present note aims to exhibit a simple method for finding the total number of the subgroups contained in an arbitrary abelian group $G$ of order $g$. It will be shown that this problem can be reduced to a determination of the number of ways in which the first $l$ independent generators of highest order of a given prime power abelian group can be selected. Hence we shall first consider this question. Therefore we shall assume for the present that $g$ is of the form $p^{m}, p$ being a prime number, and that $G$ has $k$ largest invariants which are separately equal to $p^{\alpha}$. It is well known that $G$ contains a fundamental characteristic subgroup of order $p^{k}$ and of type $(1,1,1, \cdots){ }^{*}$ Each operator of order $p$ contained in this subgroup is the $p^{\alpha-1}$ power of $p^{m-k}$ operators of $G$, and no other operator of order $p$ contained in $G$ has this property. After selecting $l \leqq k$ independent generators of highest order in a set of such generators the next independent generator of this order can therefore be selected in

$$
\left(p^{k}-p^{l}\right) p^{m-k}
$$

* G. A. Miller, American Journal of Mathematics, vol. 27 (1905), p. 15. 